\title{
Dexamethasone-loaded thermo-sensitive hydrogel attenuates osteoarthritis by protecting cartilage and providing effective pain relief
}

\author{
Qi-Shan Wang ${ }^{1}$, Bing-Xin Xu' ${ }^{1}$ Kai-Jian Fan ${ }^{1}$, Yun-Shan Fan ${ }^{2}$, Hui Teng ${ }^{1}$, Ting-Yu Wang ${ }^{1}$ \\ ${ }^{1}$ Departments of Pharmacy, Shanghai Ninth People's Hospital, Shanghai Jiao Tong University School of Medicine, Shanghai, China; ${ }^{2}$ Department of \\ Orthopedics, Shanghai Tenth People's Hospital, Tongji University, Shanghai, China \\ Contributions: (I) Conception and design: QS Wang, BX Xu, TY Wang; (II) Administrative support: QS Wang, YS Fan, BX Xu; (III) Provision \\ of study materials or patients: QS Wang, KJ Fan, TY Wang; (IV) Collection and assembly of data: QS Wang, H Teng; (V) Data analysis and \\ interpretation: QS Wang, H Teng; (VI) Manuscript writing: All authors; (VII) Final approval of manuscript: All authors. \\ Correspondence to: Ting-Yu Wang. Department of Pharmacy, Shanghai Ninth People's Hospital, Shanghai Jiao Tong University School of Medicine, \\ No. 639 Zhizaoju Road, Shanghai 200011, China. Email: drtywang@163.com.
}

Background: We utilized the destabilization of medial meniscus (DMM)-induced mice to illustrate the osteoarthritis (OA) suppressing and pain-relieving effects of a novel prolonged-release intra-articular (IA)dexamethasone-loaded thermo-sensitive hydrogel (DLTH).

Methods: The effects of temperature and $\mathrm{pH}$ on DLTH formation and in vitro DLTH release profile were assessed. C57BL/6J mice were randomly divided into three groups: Ctrl group, Model group and DLTH group. The DLTH group received joint injections of $10 \mu \mathrm{L}$ DLTH $(1 \mathrm{mg} / \mathrm{kg})$ into the right knee once a week from week 2 to week 11. We performed micro-computed tomography (Micro-CT) and histological analyses of safranin O-fast green, hematoxylin and eosin, and tartrate-resistant acid phosphatase in knee joints. We also carried out immunohistochemical (IHC) staining for matrix metalloproteinase-9 (MMP-9), MMP-13, and a disintegrin and metalloproteinase with thrombospondin motifs-5 (ADAMTS-5) in cartilage and Ki-67 in synovia. Pain behavioral testing was carried out in all mice. The serum content of prostaglandin E2 (PGE2) and real-time polymerase chain reaction (PCR) of inflammatory cytokines and pain-related factors in dorsal root ganglia (DRGs) were evaluated.

Results: It took 20 minutes to form DLTH at pH 7.0 and $37^{\circ} \mathrm{C}$. The cumulative release profiles of dexamethasone (Dex) from DLTH at $37^{\circ} \mathrm{C}$ revealed a rapid release in the first $24 \mathrm{~h}$ and a sustained slow release for 7 days. In vivo study illustrated that DLTH attenuated OA bone destruction and ameliorated synovitis and progression of OA in DMM-induced mice. The chondroprotective effects of DLTH were mediated by decreased expressions of MMP-9, MMP-13, and ADAMTS-5. The results showed that IADLTH exerted pain-relieving effects in OA mice. Upregulation of nociceptive response time (NRT) and downregulations of serum PGE2, inflammatory factors, and pain-related mediators in DRGs of mice in the DLTH group were recorded.

Conclusions: Data presented in this study elucidated that DLTH exhibited a long and lasting Dex release and it is a potential sustainable drug delivery system (DDS) to treat OA locally. IA-DLTH injection exerted chondroprotective and pain-relieving effects in DMM-induced arthritis. The involvement of MMP-9, MMP-13, ADAMTS-5, and inflammatory and pain-related factors, may account for the suppression of OA progression and pain.

Keywords: Cartilage protection; dexamethasone-loaded thermo-sensitive hydrogel (DLTH); pain relief; osteoarthritis 
Submitted Feb 09, 2021. Accepted for publication Jun 11, 2021.

doi: 10.21037/atm-21-684

View this article at: https://dx.doi.org/10.21037/atm-21-684

\section{Introduction}

Osteoarthritis (OA) is one of the most prevalent chronic degenerative arthritic diseases. The major pathological abnormalities of $\mathrm{OA}$ include cartilage degeneration, remodeling of subchondral bone, and synovial inflammation; these abnormalities may be primarily responsible for the structural damages of OA (1). A disturbance in the equilibrium between anabolism and catabolism of cartilage in OA has also been established, particularly an increase in catabolism (2). Pain is another hallmark of knee OA, mainly due to cartilage destruction and subchondral bone remodeling. Although OA-induced pain often affects joints, widespread sensitization has also been reported in many patients (3).

The most common arthritis interventions involve oral anti-inflammatory drugs, intra-articular (IA) medications and surgical treatments. Multiple lines of evidence have supported a crucial role for glucocorticoids (GCs) in OA treatment, and IA injection of GCs is the mainstay of OA management. With a potency of approximately 20 times greater than naturally-occurring hydrocortisone, dexamethasone (Dex) remains widely recommended as a significant complementary medication in OA treatment $(4,5)$. Huebner et al. have reported that administration of IA Dex is chondroprotective in a rabbit model of OA (6). Since the systemic administration of Dex occasionally causes allergies or toxic adverse effects, the application of IA Dex injection is the preferred choice of local administration because of its unique advantages. For example, IA injection can increase bioavailability, decrease off-target effects, and reduce overall drug costs. However, IA injection still presents the issue of rapid clearance of therapeutic medications (7). Lymphatic drainage and rapid diffusion from synovial capillaries have significantly shortened the drug's remains in the joint cavity. Moreover, IA medications are usually in free form, leading to the rapid escape from the joint cavity into the systematic circulation and patients needing frequent IA injections that can occasionally cause side effects. Therefore, improvements for IA injections are urgently needed.

Hence, using chitosan-glycerin-borax as a carrier, a novel prolonged-release IA formulation of dexamethasoneloaded thermo-sensitive hydrogel (DLTH) was developed.
As an in situ drug delivery system (DDS), this in situ forming hydrogel is applied as a solution that undergoes gelation in the joint cavity due to changes in temperature or $\mathrm{pH}$ (8). The hydrogel responds to temperature and the initial liquid formulation flows easily and fills the target site of any shape. The liquid formulation can be kept at room temperature before IA injection but undergoes gelation when the temperature reaches $37^{\circ} \mathrm{C}$ (normal body temperature). The soft and wetting surface of the gel has an excellent affinity to tissues and reduced stimulation to the surrounding joint cavity, which implies that the thermallyresponsive hydrogel has preferable biocompatibility. The thermally-responsive hydrogel-DLTH remains liquid at room temperature but turns into a gel station at $37^{\circ} \mathrm{C}$ in about 20 min (9). Utilization of DLTH provides enhanced medication retention time in the joint cavity, achieves high and sustained drug concentration at the desired site, and eliminates potential adverse effects with low-frequency therapy. These factors lead to improved therapeutic indices and fewer adverse effect risks of Dex (10). DLTH may provide a promising treatment of arthritis.

Destabilization of medial meniscus (DMM) is one of the most widely used surgically induced OA models. In this study, we utilized DMM-induced mice to illustrate the OA suppressing and pain-relieving effects of IA-DLTH in studies of OA progression and OA-related pain research. In doing so, we uncovered the underlying mechanisms of the thermally-responsive hydrogel in OA suppression and effective pain relief.

We present the following article in accordance with the ARRIVE reporting checklist (available at https://dx.doi. org/10.21037/atm-21-684).

\section{Methods}

\section{Preparation of DLTH}

Preparation of DLTH was shown in Figure 1. To yield a final volume proportion of glycerin:chitosan at 3:10, chitosan was dissolved in $1 \%$ acetic acid and the appropriate amount of glycerin was added. To achieve a final concentration of $2.5 \mathrm{mg} / \mathrm{mL}$ Dex at $\mathrm{pH}=7$, a borax solution and Dex were mixed with the glycerin:chitosan mixture prepared above. To 


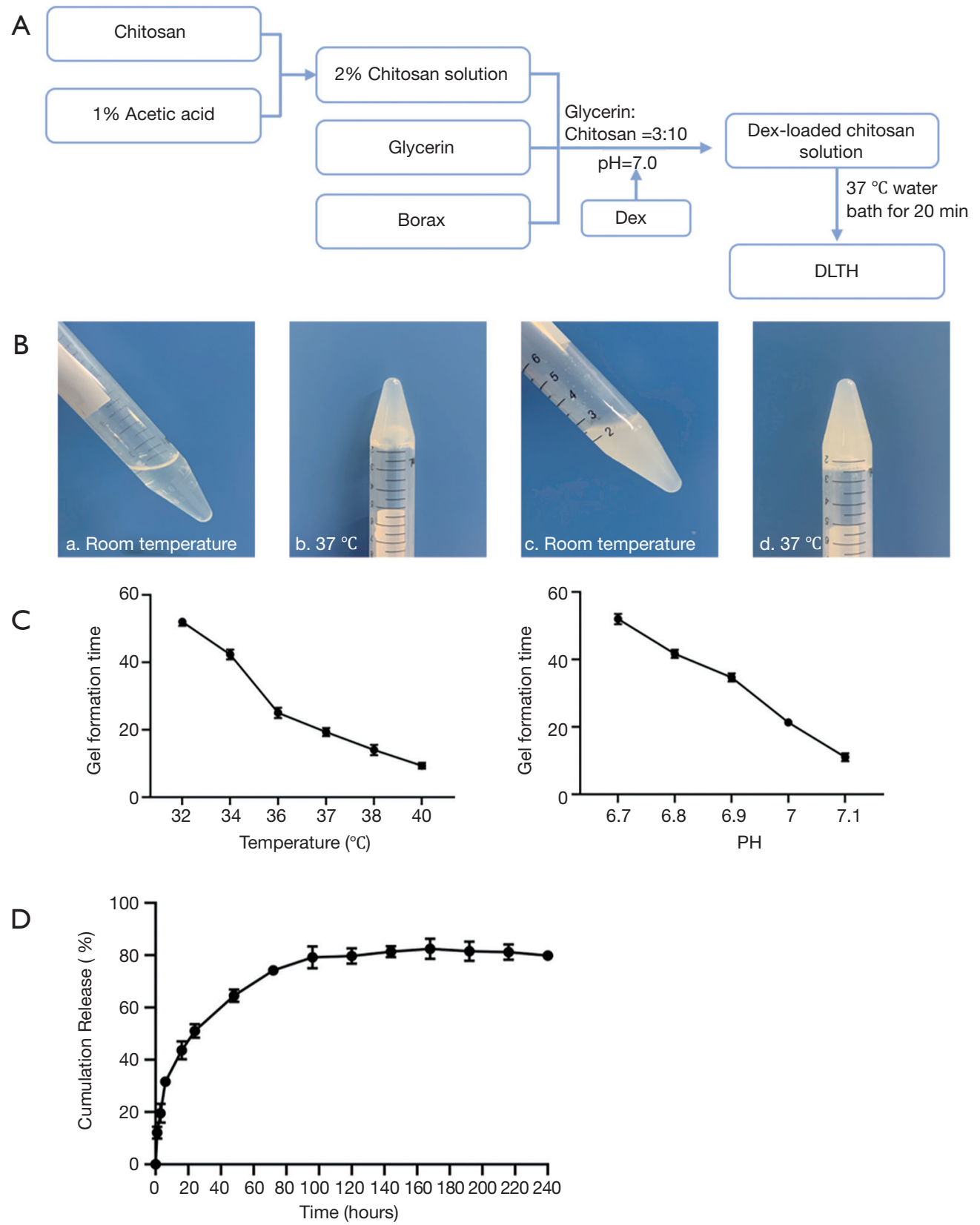

Figure 1 Profile of DLTH. (A) Flowchart of the preparation of DLTH. (B) The morphology of chitosan blank hydrogels at different temperatures (a,b), and photographs of DLTH in a soluble state at room temperature and in the gel state at $37{ }^{\circ} \mathrm{C}(\mathrm{c}, \mathrm{d})$. (C) Effects of temperature and $\mathrm{pH}$ on DLTH formation. (D) In vitro release of DLTH in PBS at $37^{\circ} \mathrm{C}$. All data were expressed as mean \pm SEM. DLTH, dexamethasone-loaded thermo-sensitive hydrogel; PBS, phosphate-buffered solution; SEM, standard error of the mean.

obtain an injectable thermo-sensitive chitosan-glycerol-borax hydrogel, the freshly prepared final DLTH mixture was bathed in $37^{\circ} \mathrm{C}$ water for 20 minutes (Figure $1 A$ ). A flowable liquid was observed at room temperature and a semi-solid gel state was observed at $37^{\circ} \mathrm{C}$ (Figure 1B) (11).

\section{DLTH formation time and in vitro DLTH release evaluation}

Six tubes of the freshly prepared final DLTH solution were formulated according to the method described above. To obtain an injectable DLTH, six tubes $(5 \mathrm{~mL})$ of DLTH 
solution were bathed in a constant temperature water bath at $32,34,36,37,38$, and $40{ }^{\circ} \mathrm{C}$, respectively. To obtain DLTH, five tubes $(5 \mathrm{~mL})$ of DLTH solution were formed, ranging from $\mathrm{pH} 6.7$ to $\mathrm{pH} 7.1$, in $37^{\circ} \mathrm{C}$ water baths. No flowable fluid is a sign of successful gel formation, and the time required for DLTH formation was recorded. All assays were repeated at least three times.

Successfully prepared DLTH $(5 \mathrm{~mL})$ and $15 \mathrm{~mL}$ of

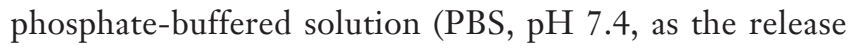
medium) were added to the upper part of the gel. The DLTH with PBS buffer was placed in a constant temperature shaker at $37^{\circ} \mathrm{C}$. At specific time points $(1,3,6,12,24,48,72$, $96,120,144,168,192,216$ and $240 \mathrm{~h}$ ), $5 \mathrm{~mL}$ of the buffer sample was removed, and another $5 \mathrm{~mL}$ of fresh-release medium was added to maintain a constant volume. The released samples were analyzed by UV spectrophotometry at $242 \mathrm{~nm}$, and the in vitro cumulative release was calculated.

\section{Animals and induction of the mouse DMM OA model}

Eighteen male C57BL/6J mice aged 10 weeks were purchased from Shanghai SLAC Laboratory Animal Company (Shanghai, China). Ethical approval was obtained from the Animal Ethical Committee of Shanghai Ninth People's Hospital (approval number: HKDL [2015] 16). Adult mice were fed with food and water ad libitum, and the in vivo experiments were in accordance with the Code of Practice for the Housing and Care of Animals Used in Scientific Procedures and the ARRIVE (Animals in Research: Reporting In Vivo Experiments) guidelines $(12,13)$. The mice were randomly divided into three groups: Ctrl group, Model group, and DLTH group. Experimental OA was established by destabilization of the medial meniscus (DMM) surgery: mice in the Model group and DLTH group received DMM of the right knee, whereas those in the Ctrl group underwent sham operations (14). The DLTH group was subjected to joint injections of $10 \mu \mathrm{L}$ DLTH ( $1 \mathrm{mg} / \mathrm{kg}$ Dex per mouse) into the right knee once a week from week 2 to week 11 , while the Ctrl and Model groups were given an equal volume of saline into the same knee (Figure 2A).

\section{Pain behavioral testing}

The latency of hot plate nociception assessed thermal hyperalgesia. Before the assessment, the mice were allowed to acclimate for more than 20 minutes. Each mouse was then placed on a metal-hot plate (Columbus Instruments) set at $55^{\circ} \mathrm{C}$ and observed for no more than 45 seconds.
The withdrawal of any paw was regarded as a nociceptive response, and the nociceptive response time (NRT) was recorded (15). Each animal was tested at least three times, and the average NRT was calculated. All behavioral pain tests were conducted in the morning every other week for more than 12 weeks.

\section{Micro-computed tomography (Micro-CT) scanning}

At the end of week 12, all mice were anesthetized with sodium pentobarbital intraperitoneally $(40 \mathrm{mg} / \mathrm{kg}$ ) and then sacrificed by cervical dislocation. The knee joints of all groups were dissected and fixed in 4\% paraformaldehyde. The mouse samples were scanned with Micro-CT (Siemens Co., Knoxville, Tennessee, USA), which was performed at the following parameters: voltage of $70 \mathrm{kV}$, an electric current of $114 \mu \mathrm{A}$, and resolution of $10 \mu \mathrm{m}$ per pixel. Threedimensional structures were analyzed, including bone volume (BV), bone volume/total tissue volume (BV/TV), trabecular number (Tb.N), trabecular thickness (Tb.Th), and trabecular separation (Tb.Sp).

\section{Histological analyses}

Knee joint specimens were decalcified in 10\% EDTA and then embedded in paraffin. For microstructure observation, 5 - $\mu \mathrm{m}$-thick sagittal sections were prepared and stained with safranin $\mathrm{O}$-fast green $(\mathrm{S} \& \mathrm{~F})$, hematoxylin and eosin (H\&E), and tartrate-resistant acid phosphatase (TRAP). Osteoclast and osteoblast activity in the knee joint was assessed with TRAP and H\&E staining to detect the bone destruction of the joint (16). Articular cartilage degradation and destruction were quantified using Osteoarthritis Research Society International (OARSI) score, with a total score of 24. Higher scores indicated more severe destruction of cartilage (17).

The synovial histological analyses were also accomplished as previously described $(18,19)$. Hyperplasia was scored from 0 to 3 as follows: a score of 0 denoted normal cell structure, and a score of 3 suggested severe synovial hyperplasia. Synovial inflammation was scored on a scale from 0 to 3 , where 0 signified no evidence of inflammation or synovial lining cell hyperplasia, and 3 indicated severe synovial inflammation with articular cartilage damage.

We also performed immunohistochemical (IHC) staining for matrix metalloproteinase-9 (MMP-9), MMP-13, a disintegrin and metalloproteinase with thrombospondin motifs-5 (ADAMTS-5) and Ki-67 (Abcam, United Kingdom; dilution 1:100). The percentage of positively stained and 


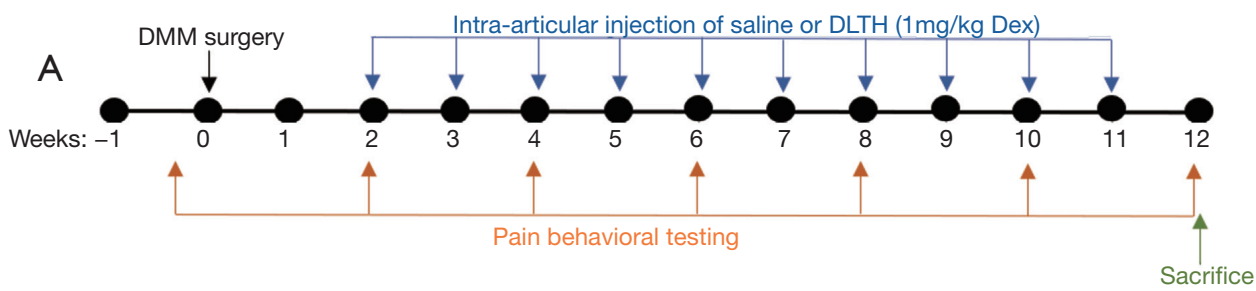

B
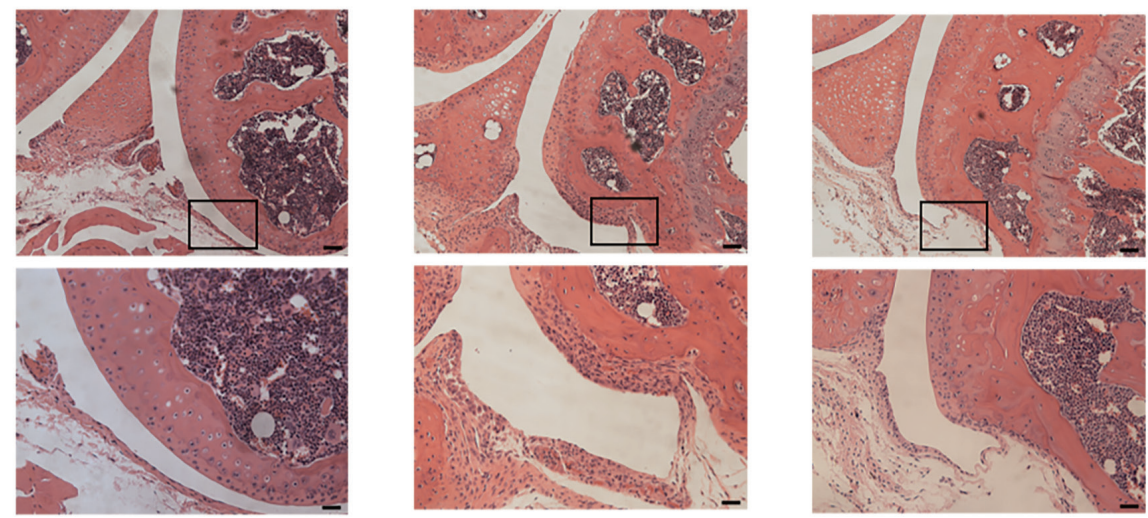

C
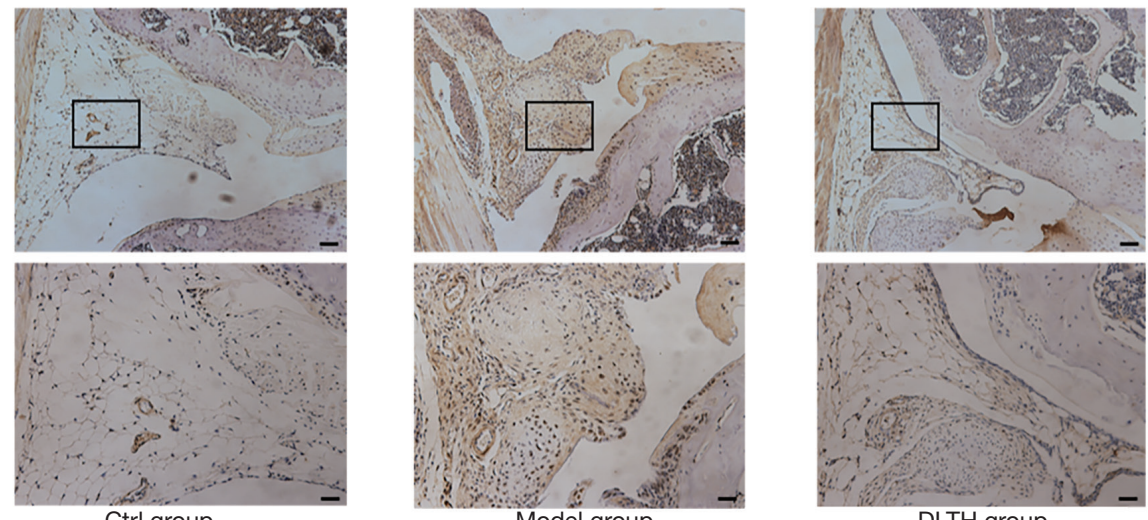

D

Ctrl group

Model group

DLTH group

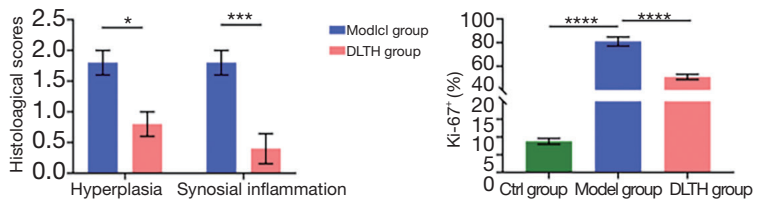

Figure $2 \mathrm{H} \& \mathrm{E}$ and immunohistochemical staining of DLTH treatment in DMM-induced osteoarthritis. (A) Schematic of the experimental study design. Three groups of male C57BL/6J mice were studied: Ctrl group, Model group and DLTH group. Mice in the Model and DLTH groups received DMM surgery on the right hind knees at 10 weeks of age, while mice in the Ctrl group underwent sham operations in the same knee. Mice in the DLTH group were subjected to joint injection of $10 \mu \mathrm{L}$ DLTH $(1 \mathrm{mg} / \mathrm{kg}$ Dex per mouse) into the right knee once a week from week 2 to week 11 , while mice in the $\mathrm{Ctrl}$ and Model groups were given an equal volume of saline in the same knee. The timelines for DMM surgery, IA-DLTH injection, and behavioral pain testing are shown. (B) Histological analyses of synovial joints in DLTH-treated OA mice. Representative photographs of mice knee joints stained with H\&E were displayed. Scale bar, $200 \mu \mathrm{m}$ (upper panel) and $100 \mu \mathrm{m}$ (lower panel). Black boxes represented the magnification area in the corresponding figure below. (C) Ki-67 staining of synovia in DLTH-treated OA mice. Representative photographs of mice knee joints stained with Ki-67 are shown. Scale bar, $200 \mu \mathrm{m}$ (upper panel) and $100 \mu \mathrm{m}$ (lower panel). Black boxes represented the magnification area in the corresponding figure below. (D) Quantification of hyperplasia and synovial inflammation using semiquantitative histology scoring system; Ki-67 positive cells in the synovia are displayed. All data were expressed as mean $\pm \mathrm{SEM}$. ${ }^{*} \mathrm{P}<0.05 ;{ }^{* * *} \mathrm{P}<0.001 ;{ }^{* * *} \mathrm{P}<0.0001$. Ctrl, control; DLTH, dexamethasone-loaded thermo-sensitive hydrogel; DMM, destabilization of the medial meniscus; H\&E, hematoxylin and eosin; IA, intra-articular; OA, osteoarthritis; SEM, standard error of the mean. 
Table 1 Sequences of specific primers used in RT-qPCR

\begin{tabular}{|c|c|c|}
\hline Primers & \multicolumn{2}{|c|}{ Sequences } \\
\hline$\beta$-actin & 5'-GTGACGTTGACATCCGTAAAGA-3' & 5'-GCCGGACTCATCGTACTCC-3' \\
\hline IL-1 $\beta$ & 5'-GAAATGCCACCTITTGACAGTG-3' & 5'-TGGATGCTCTCATCAGGACAG-3' \\
\hline IL-6 & 5'-CTGCAAGAGACTTCCATCCAG-3' & 5'-AGTGGTATAGACAGGTCTGTTGG-3' \\
\hline TNF- $\alpha$ & 5'-CAGGCGGTGCCTATGTCTC-3' & 5'-CGATCACCCCGAAGTTCAGTAG-3' \\
\hline coX-2 & 5'-TTCCAАTCCATGTCAAAACCGT-3' & 5'-AGTCCGGGTACAGTCACACTT-3' \\
\hline EP1 & 5'-CTTAACCTGAGCCTAGCGGAT-3' & 5'-ATGTGCCATTATCGCCTGTTG-3' \\
\hline EP2 & 5'-CAGCTCGGTGATGTTCTCGG-3' & 5'-GAGCACCAATTCCGTTACCAG-3' \\
\hline NGF & 5'-CCAGTGAAATTAGGCTCCCTG-3' & 5'-CCTTGGCAAAACCTITATTGGG-3' \\
\hline TRPV1 & 5'-CCCGGAAGACAGATAGCCTGA-3' & 5'-TTCAATGGCAATGTGTAATGCTG-3' \\
\hline Nav 1.3 & 5'-CAGACCATGTGCCTTATTGTGT-3' & 5'-CCGCGATCTGGAGGTTGTT-3' \\
\hline Nav 1.7 & 5'-TGGATTCCCTTCGTTCACAGA-3' & 5'-GTCGCAGATACATCCTCTTGTTI-3' \\
\hline BDNF & 5'-TCATACTTCGGTTGCATGAAGG-3' & 5'-AGACCTCTCGAACCTGCCC-3' \\
\hline NPY & 5'-ATGCTAGGTAACAAGCGAATGG-3' & 5'-TGTCGCAGAGCGGAGTAGTAT-3' \\
\hline
\end{tabular}

RT-qPCR, real-time quantitative polymerase chain reaction; NGF, nerve growth factor; TRPV1, transient receptor potential vanilloid 1; BDNF, brain-derived neurotrophic factor; NPY, Neuropeptide Y; IL-1 $\beta$, interleukin-1 $\beta$; PGE receptor subtype 1; EP1, TNF- $\alpha$, tumor necrosis factor- $\alpha$; COX-2, cyclo-oxygenase 2.

total chondrocytes in the articular cartilage per specimen was calculated as previously described (20). Expression levels of MMP-9, MMP-13, and ADAMTS-5 in the entire articular cartilage, and Ki-67 expression in synovia, were calculated by two blinded independent observers. Images were obtained with a Zeiss Axio Imager light microscope.

\section{Real-time quantitative polymerase chain reaction (RT- qPCR)}

At the end of 12 weeks, the L4-L6 dorsal root ganglions (DRGs) of all mice were collected and minced in TRIzol Reagent (Ambion, Shanghai, China) for total RNA extraction. Total RNA was reverse transcripted using a cDNA Reverse Transcription Kit (Takara Bio, Tokyo, Japan) following the manufacturer's protocol. RT-qPCR was conducted using a 7500 Real-Time PCR System (Thermo Fisher Scientific) with specific primers. Gene expression values were normalized to $\beta$-actin, and quantifications of gene expressions were calculated adopting the $2^{-\Delta \Delta C T}$ method. All analyses were repeated three times. See Table 1.

Enzyme-linked immunosorbent assay (ELISA) detection of serum prostaglandin E2 (PGE2)

To obtain plasma, blood was sampled from mice eyes and centrifuged at 3,600 rpm for 10 minutes. Serum levels of PGE2 were measured using commercial ELISA kits (Elabscience, Wuhan, China). A microplate reader-generated optical density (OD) values at $450 \mathrm{~nm}$. All experiments were conducted in duplicate and serum contents were calculated by reference to standard curves.

\section{Statistical analysis}

All statistical analyses were obtained from more than three experiments and performed using SPSS20.0 software. All data are shown as mean \pm standard error of the mean (SEM). 
Comparison significance tests were conducted via oneway ANOVA followed by Turkey multiple test or Student's $t$-test. $\mathrm{P}$ values less than 0.05 were considered statistically significant.

\section{Results}

\section{Effects of temperature and $\mathrm{pH}$ on DLTH formation}

The Dex-loaded chitosan gel formed a semisolid-state when the temperature reached $32{ }^{\circ} \mathrm{C}$, with higher temperatures reducing the gelation time. It took 20 minutes to form a semi-solid gel at $37^{\circ} \mathrm{C}$, indicating that in the joint cavity, the DLTH gel could be formed in 20 minutes.

The Dex-loaded chitosan gel was formed in a nearneutral environment, ranging from $\mathrm{pH} 6.7$ to $\mathrm{pH}$ 7.1. In the $\mathrm{pH}$ range of 6.7 to 7.1 , the gel formation time decreased as the system's $\mathrm{pH}$ increased. The turbidity of the gel system at $\mathrm{pH} 7.1$ was relatively high, and filaments of precipitates were occasionally observed. Therefore, DLTH in this study was conducted at $\mathrm{pH}$ 7.0. See Figure 1C.

\section{In vitro Dex release from $\mathrm{DLTH}$}

To determine the in vitro Dex release from DLTH, the cumulative release (\%) of DLTH was measured at various time points. Figure $1 C$ displays the cumulative release profiles of Dex from DLTH at $37^{\circ} \mathrm{C}$, reflecting a sustained slow release for 7 days. About $80 \%$ of Dex was released from DLTH in the first 7 days and there was almost no release in the following 3 days. The release profiles of DLTH exhibited a rapid release in the first $24 \mathrm{~h}$ and a prolonged release for $168 \mathrm{~h}$ (Figure 1D).

\section{DLTH ameliorates synovitis in $\mathrm{OA}$ mice}

There was no mouse death throughout the entire experiment. Histological observations and semiquantitative scores of OA, in terms of synovial hyperplasia and synovial inflammation, were significantly more obvious in the Model group. DMM surgery resulted in increased thickness of the synovial membrane, while DLTH protected against synovial hyperplasia and inflammation in OA mice (Figure 2B). There was no sign of inflammatory activity in the Ctrl group.

Synovial cell proliferation was increased in the inflamed synovia as determined by immunohistochemistry of the nuclear marker Ki-67. The expression of proliferation-specific $\mathrm{Ki}-67$ antigen in the synovial membrane was increased in the Model group compared with that in the Ctrl group. However, following treatment with IA-DLTH injection, there were significantly decreased expression levels of Ki-67 in synovial lining cells in the DLTH group compared with those in the Model group (Figure 2C). Thus, there may be a positive correlation between immunohistochemistry results and the degree of synovitis. See Figure 2D.

\section{DLTH attenuated $\mathrm{OA}$ bone destruction}

As shown in Figure 3, DMM surgery-induced elevated bone resorption in the subchondral bone, as displayed by advanced osteolysis. However, the results signified that DLTH therapy largely reduced bone loss. Statistically, sagittal views of the joints demonstrated that $\mathrm{BV}$ in the Model group was clearly down-regulated but was remarkably restored after DLTH joint injection. The parameters of micro-CT, involving BV/TV, Tb.N, Tb.Th, and Tb.Sp, also revealed that DLTH markedly ameliorated bone destruction in vivo. See Figure 3.

As shown by TRAP and H\&E staining in the trabecular bone region, IA-DLTH suppressed DMM-induced generation of osteoclasts and increased the formation of osteoblasts. Compared with the Ctrl group, mice in the OA Model group demonstrated significantly higher osteoclast counts and notably lower formation of osteoblasts. Treatment with DLTH restrained osteoclast numbers and improved osteoblast formation in this model, thereby attenuating OA progression. See Figure 4.

\section{DLTH slowed OA progression by protecting cartilage in DMM-induced mice}

$\mathrm{H} \& \mathrm{E}$ and $\mathrm{S} \& \mathrm{~F}$ staining revealed the loss of chondrocytes and degeneration of the cartilage layer in DMM-induced mice. However, in DLTH-treated DMM mice, the decreased thickness of cartilage was largely inhibited, evidenced by prominently decreased OARSI scores. These results suggested that DLTH might slow the progression of OA by protecting cartilage. See Figure 5.

\section{Expressions of MMP-9, MMP-13 and ADAMTS-5 mediated the chondroprotective effects of DLTH in DMM- induced mice}

Expressions of MMP-9, MMP-13 and ADAMTS-5 in the Model group were highly upregulated by DMM surgery, whereas DLTH inhibited catabolic marker gene expressions 

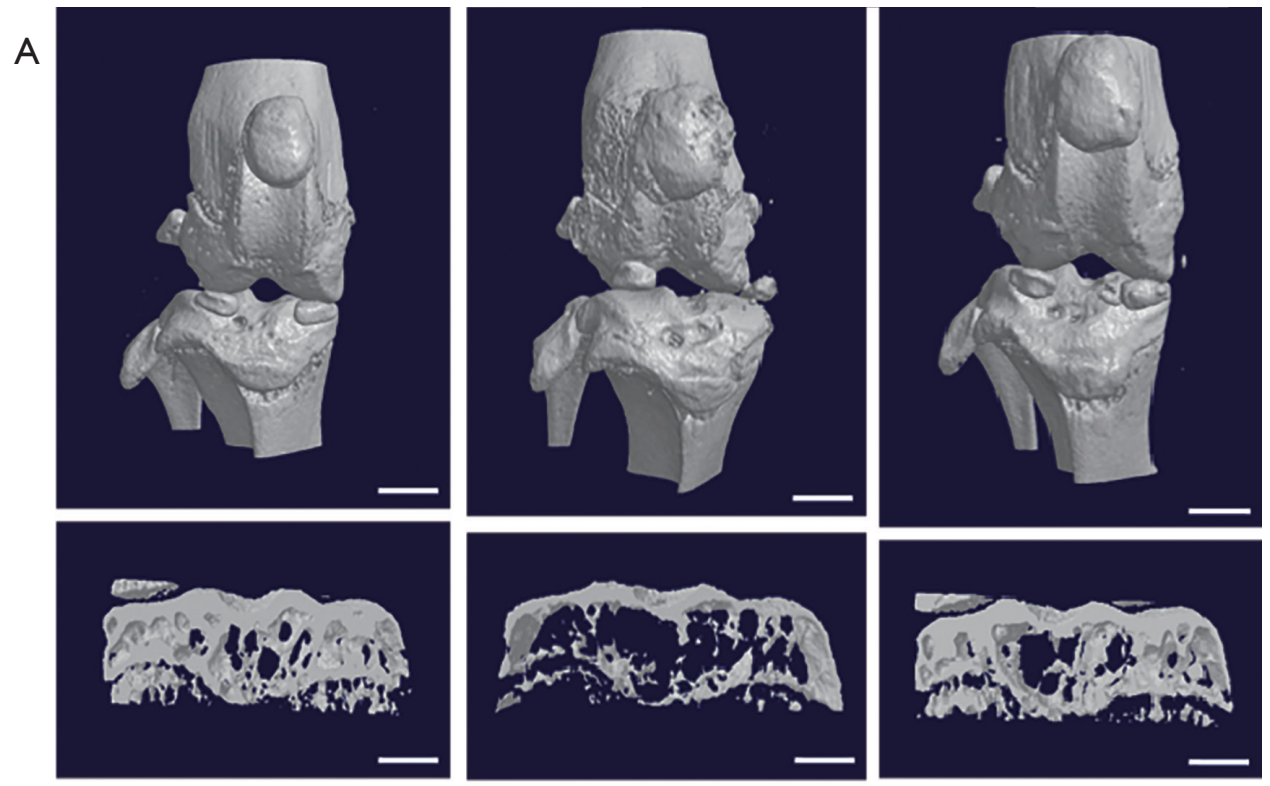

Ctrl group

Model group

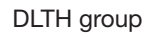

B
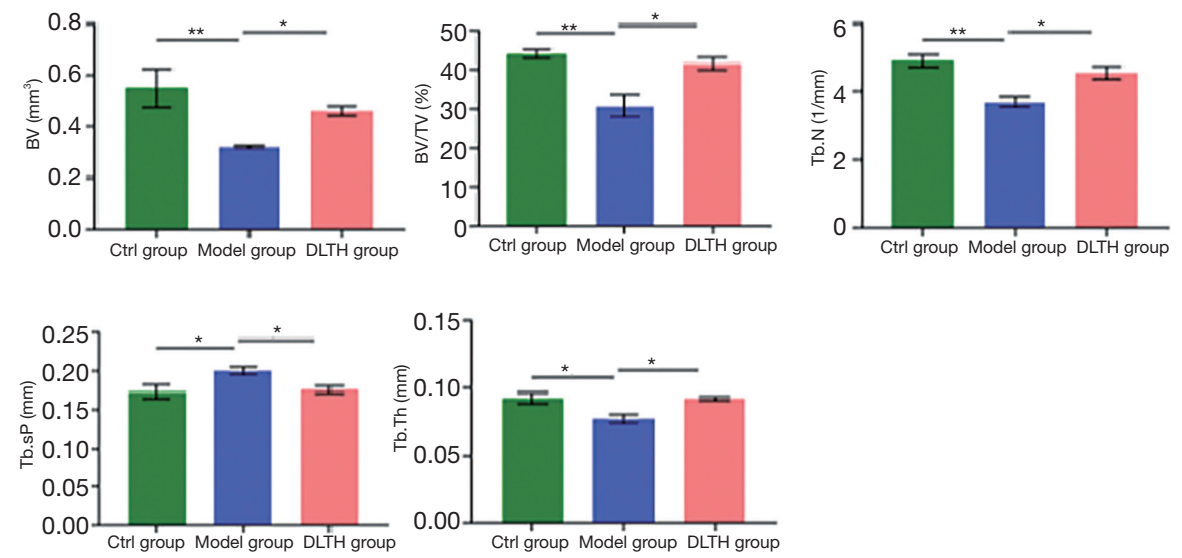

Figure 3 Micro-CT analysis of DLTH-treated OA mice induced by DMM surgery. (A) Three-dimensional Micro-CT images of frontal views of knee joints following DLTH treatment and the medial compartment of the subchondral bone of the sagittal views are shown. Scale bar: $1 \mathrm{~mm}$. (B) Quantitative analyses of BV, BV/TV, Tb.N, Tb.Sp, and Tb.Th are also displayed. All data were expressed as mean \pm SEM. *P $<0.05$; ${ }^{* *} \mathrm{P}<0.01$. BV, bone volume, BV/TV; bone volume/total tissue volume; DLTH, dexamethasone-loaded thermo-sensitive hydrogel; DMM, destabilization of the medial meniscus; Micro-CT, micro-computed tomography; OA, osteoarthritis; SEM, standard error of the mean.

in the cartilage of DMM-induced mice. These findings suggested that DLTH exerted potent suppressive effects in the catabolic responses of OA cartilage. See Figure 6.

\section{Foint injection of DLTH exerts pain-relieving effects in OA mice}

To assess the pain-reducing effects of DLTH, the hot plate method was performed in all groups. We found that IA-DLTH exerted analgesic actions in terms of the NRT. Compared with the Ctrl group, the NRT of the Model group was considerably decreased. However, following 10 weeks of DLTH treatment, the NRT of OA mice in the DLTH group gradually increased as evidenced by a markedly longer paw flick response time. This enhancement of NRT indicated an increased pain threshold. Therefore, we concluded that reduced NRTs were exhibited in OA mice; however, DLTH exerted pain-relieving effects in terms of thermal nociceptive pain. See Figure $7 A$. 
A
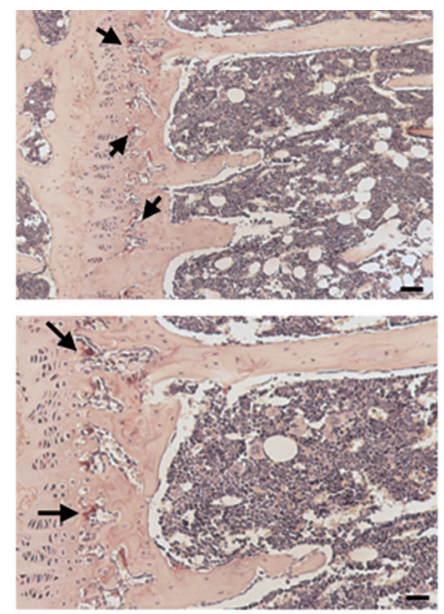

B
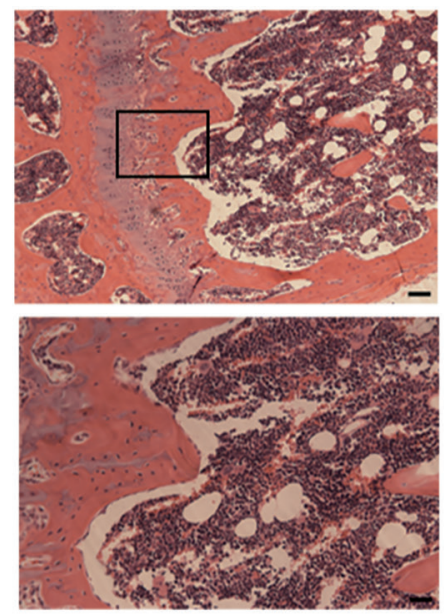

C

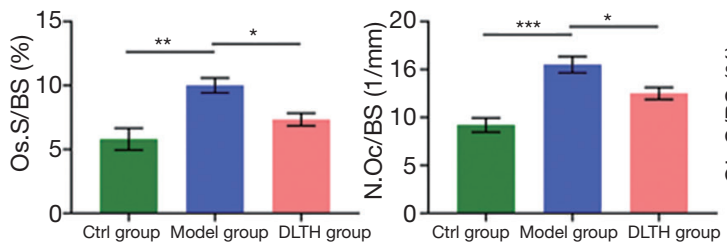

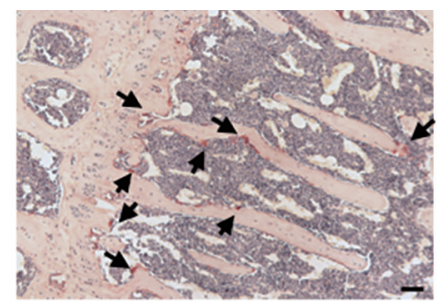
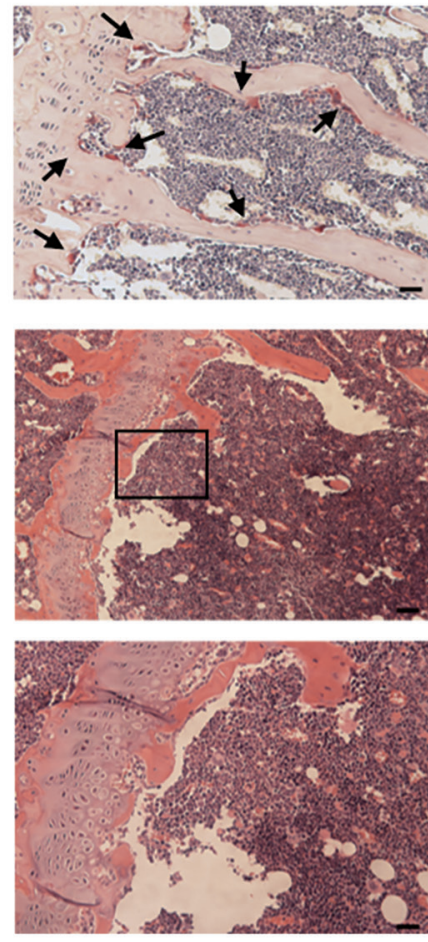

Model group
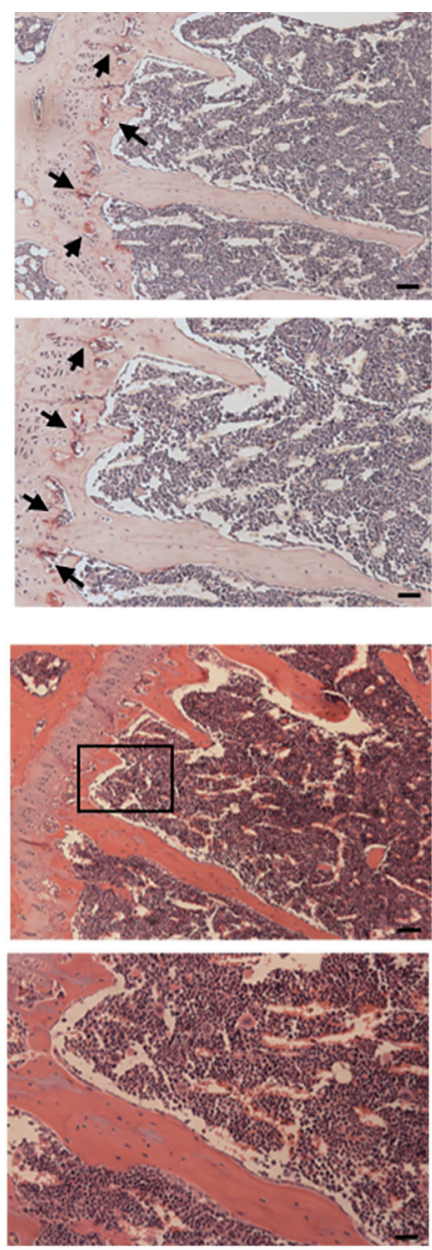

DLTH group

Figure 4 Staining analyses of DLTH treatment in DMM-induced osteoarthritis in mice. Osteoclasts and osteoblasts in subchondral bone regions were stained with TRAP (A) and H\&E (B) staining, respectively. Scale bar: $200 \mu \mathrm{m}$ (upper panel) and $100 \mu \mathrm{m}$ (lower panel). Black boxes represented the magnification area in the corresponding figure below. (C) Quantitative analyses of osteoclasts and osteoblasts counts. Black arrows indicate osteoclasts. All data were expressed as mean $\pm \mathrm{SEM}$. ${ }^{*} \mathrm{P}<0.05 ;{ }^{* *} \mathrm{P}<0.01 ;{ }^{* * *} \mathrm{P}<0.001 ;{ }^{* * *} \mathrm{P}<0.0001$. DLTH, dexamethasone-loaded thermo-sensitive hydrogel; DMM, destabilization of the medial meniscus; H\&E, hematoxylin and eosin; SEM, standard error of the mean; TRAP, tartrate resistant acid phosphatase.

\section{IA-DLTH depresses serum PGE2 and expressions of pain- related mediators in DRGs}

ELISA detected the serum levels of PGE2 in all mice. Serum PGE2 concentrations of the Ctrl, Model, and DLTH groups were $95.84 \pm 14.98 \mathrm{pg} / \mathrm{mL}, 334.88 \pm 38.21 \mathrm{pg} / \mathrm{mL}$, and $89.83 \pm 39.55 \mathrm{pg} / \mathrm{mL}$, respectively (Figure $7 B$ ). The diminished production of blood serum PGE2 with IADLTH therapy demonstrated that DLTH administration might mitigate $\mathrm{OA}$ pain, consistent with the results of the 


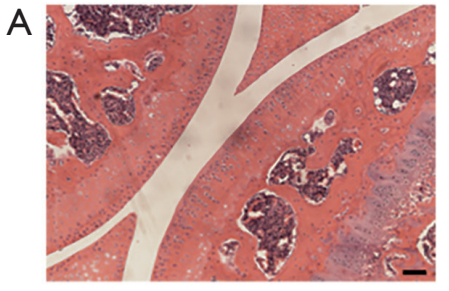

B
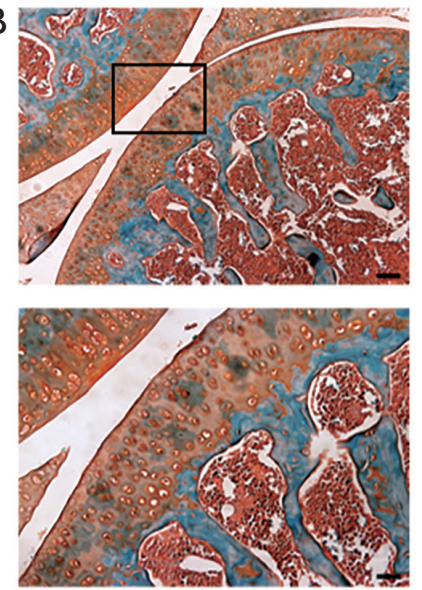

Ctrl group

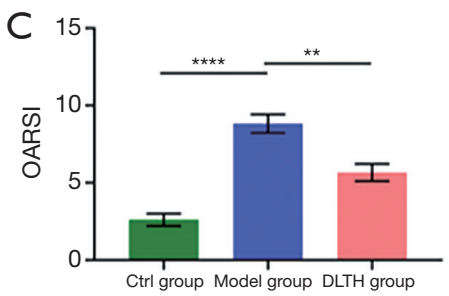

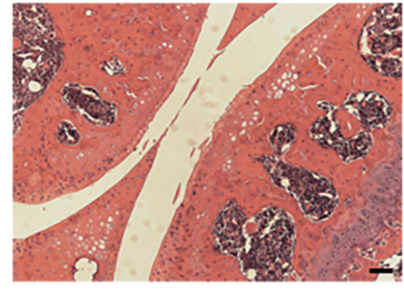
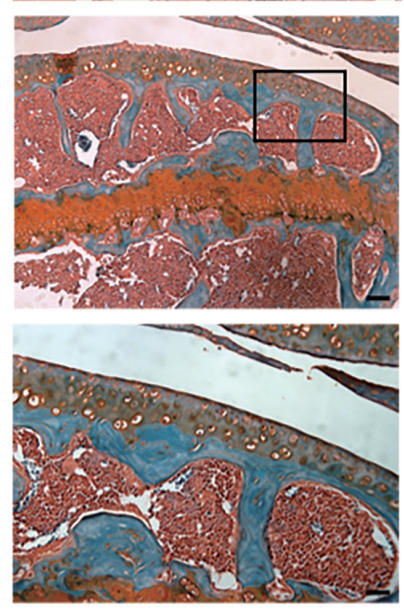

Model group
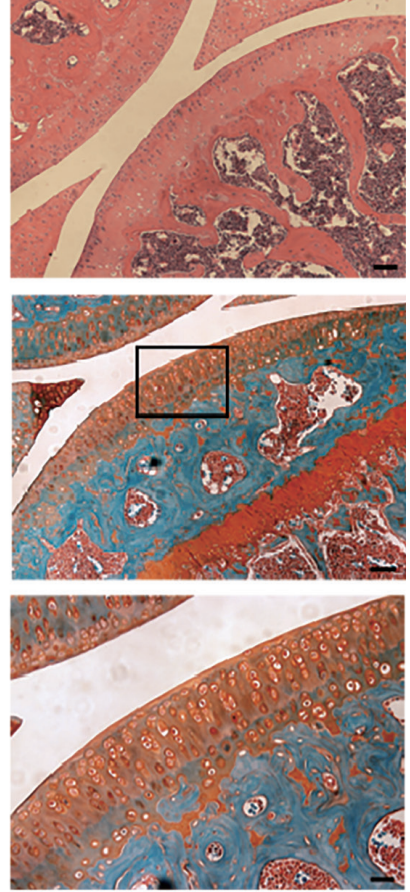

DLTH group

Figure $5 \mathrm{~S} \& \mathrm{~F}$ and H\&E staining of DLTH treatment in DMM-induced osteoarthritis. (A,B) H\&E and S\&F staining of knee joint medial compartment cartilage and subchondral bone in DMM-induced mice. Scale bar: $200 \mu \mathrm{m}$ (upper panel) and $100 \mu \mathrm{m}$ (lower panel). Black boxes represented the magnification area in the corresponding figure below. (C) Quantitative analyses of OARSI scores of all groups. All data were expressed as mean \pm SEM. ${ }^{* *} \mathrm{P}<0.01 ;{ }^{* * * *} \mathrm{P}<0.0001$. DLTH, dexamethasone-loaded thermo-sensitive hydrogel; DMM, destabilization of the medial meniscus; $\mathrm{H} \& \mathrm{E}$, hematoxylin and eosin; OARSI, Osteoarthritis Research Society International; SEM, standard error of the mean; S\&F, safranin O-fast green.

pain behavior tests.

Analyses of the expressions of pro-inflammatory genes [e.g., interleukin-1 $\beta$ (IL-1 $\beta$ ), IL-6, IL-17, tumor necrosis factor- $\alpha$ (TNF- $\alpha$ ), cyclo-oxygenase 2 (COX-2), PGE receptor subtype 1 (EP1), EP2, EP3, and EP4] in dissected DRGs were also determined (Figure $7 C$ ). Elevated mRNA expressions of IL- $1 \beta$, IL-6, IL-17, TNF- $\alpha$, COX-2, EP1, EP2, EP3, and EP4 were observed in the Model group; however, but IA-DLTH suppressed secretions of the above inflammatory mediators.

Moreover, pain-related key factors, like nerve growth factor (NGF), transient receptor potential vanilloid 1 (TRPV1), voltage-gated sodium (Nav)1.3, Nav1.7, brain- derived neurotrophic factor (BDNF), and Neuropeptide Y (NPY), were also analyzed. Remarkable upregulations of these factors were observed in the Model group, while DLTH therapy decreased the mRNA levels of the abovementioned pain-related mediators. Therefore, DLTH treatment exerted pain-relieving effects by reducing proinflammatory genes and pain-related key factor expressions in OA DRGs.

\section{Discussion}

The major pathological abnormalities in OA include cartilage degeneration, remodeling of subchondral bone, 


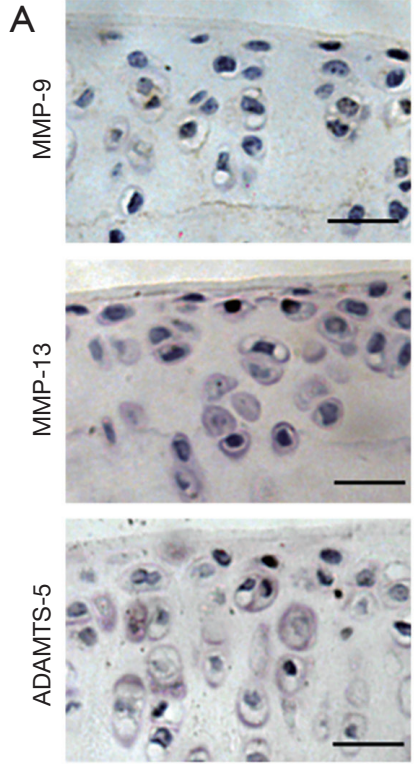

Ctrl group

B

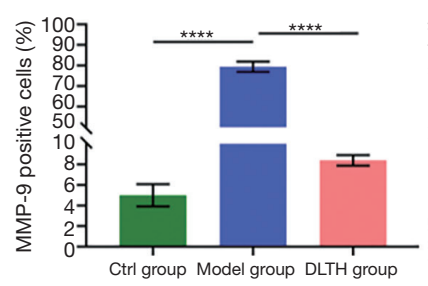

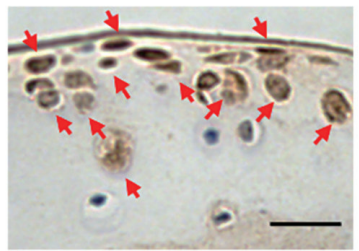
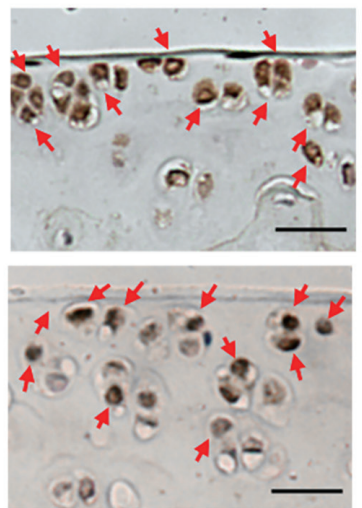

Model group

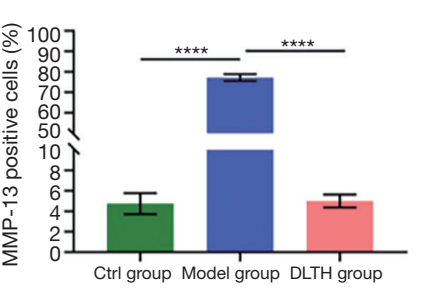

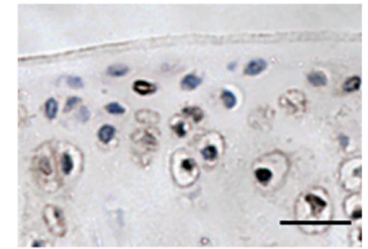
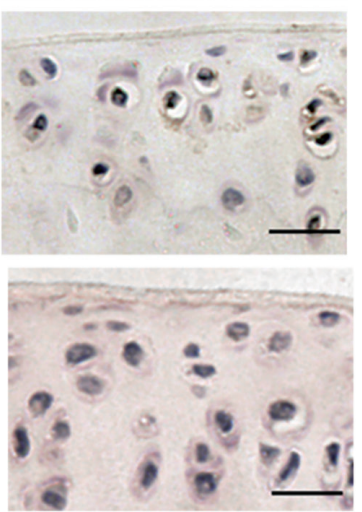

DLTH group

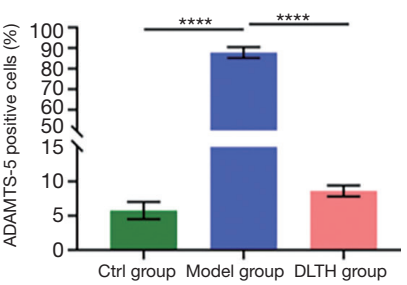

Figure 6 MMP-9, MMP-13, and ADAMTS-5 staining of chondrocytes in articular cartilage of DLTH-treated DMM-induced osteoarthritis in mice. (A) Representative photographs of mouse knee joints with immunohistochemical staining for MMP-9, MMP-13, and ADAMTS-5 are shown. (B) Quantifications of positive cells in cartilage are also displayed. Red arrows indicate positive stained chondrocytes in articular cartilage. Scale bar: $100 \mu \mathrm{m}$. All data were expressed as mean \pm SEM. ${ }^{* * * *} \mathrm{P}<0.0001$. DLTH, dexamethasone-loaded thermo-sensitive hydrogel; DMM, destabilization of the medial meniscus; SEM, standard error of the mean.

and synovial membrane inflammation (synovitis). Although the degree of inflammation in $\mathrm{OA}$ is remarkably less than that in rheumatoid arthritis, synovitis is now considered an important feature of OA that adversely affects whole joint function (21-23). Evidence-based medicine has demonstrated that acute or chronic synovitis in varying degrees was observed in OA patients (24). In the present study, we noted significant synovial hyperplasia and synovial inflammation in the Model group and found that DLTH protected against synovitis in OA mice.

To determine the possible cause of synovitis, we performed proliferative marker Ki-67 immunohistochemistry in synovial tissues. Ki-67 is widely used to identify proliferating cells in diseased synovia, including in hyperplastic synovial membranes. Pessler et al. quantified a strong association of
Ki-67 expression with a histological degree of synovitis and reported that increased $\mathrm{Ki}-67$ positive cells were found in $\mathrm{OA}$ synovial specimens in comparison with histologically normal synovial tissues (25). These results are following those of the present study. We observed increased expression of Ki-67 in synovial linings in the Model group and fewer Ki-67 positive cells in DLTH-treated OA mice. Taken together, these findings demonstrated that DLTH ameliorated synovitis in OA mice. It is believed that cartilage and bone degeneration, along with new bone formation and synovitis, lead to OA's progression and clinical symptoms (26).

Bone loss, which is another typical characteristic of OA, is correlated with the increased remodeling of subchondral bone. Multiple lines of evidence have demonstrated increased osteoclast activity and declined osteoblast 


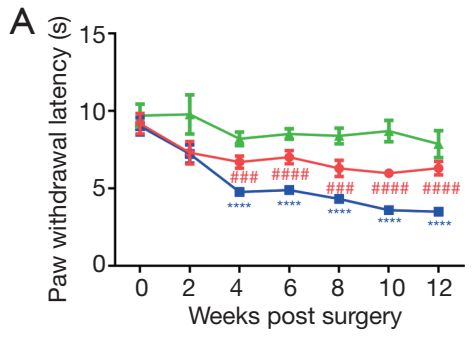

C

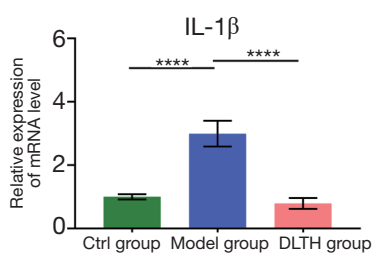

TNF- $\alpha$

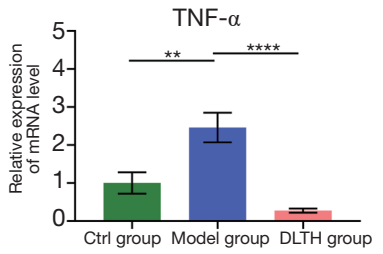

EP2

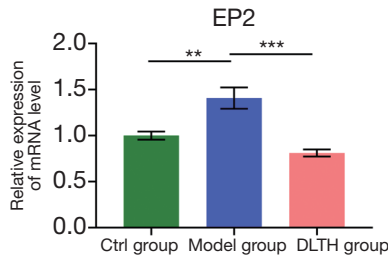

NGF
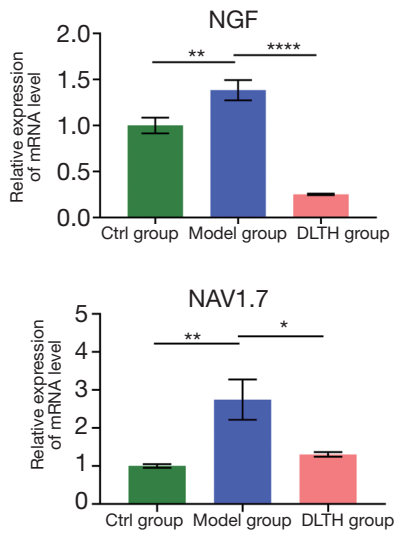

$₫$ Ctrl group

--Model group

$\rightarrow$ DLTH group
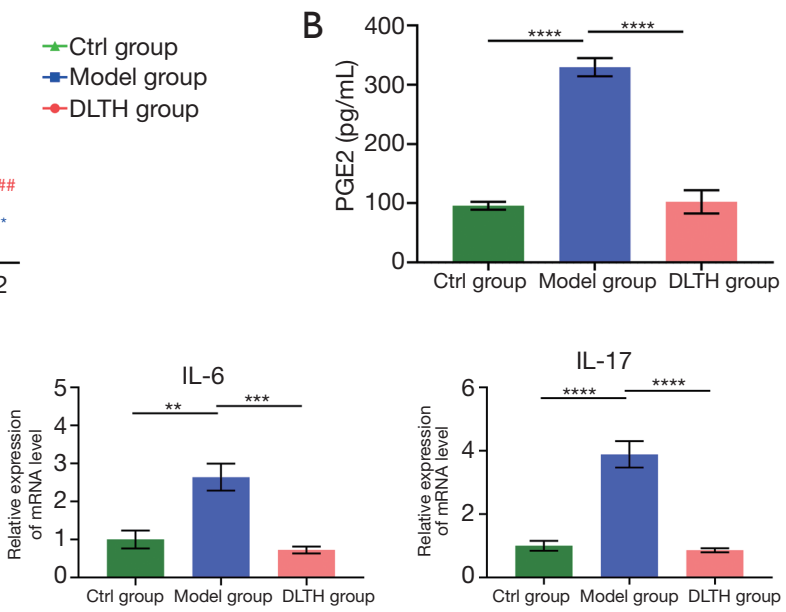

COX-2

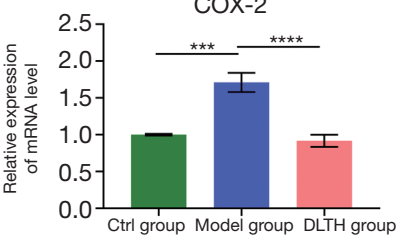

EP3
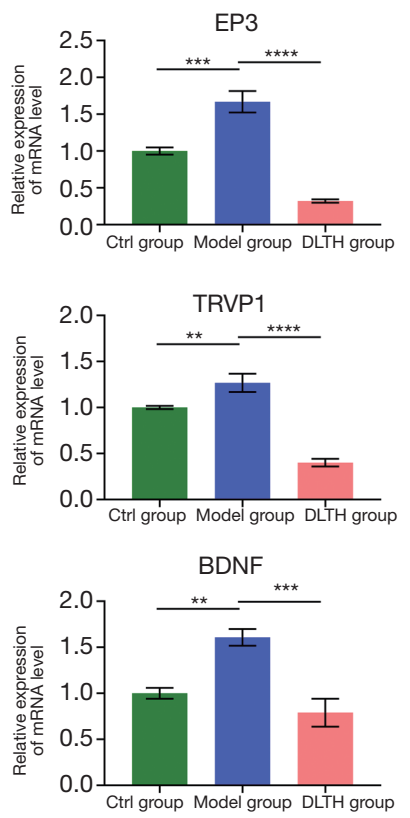
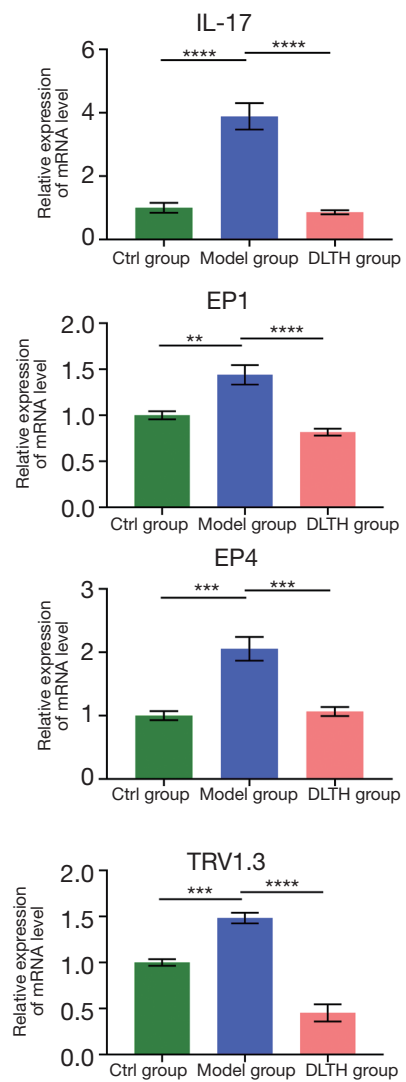

EP1

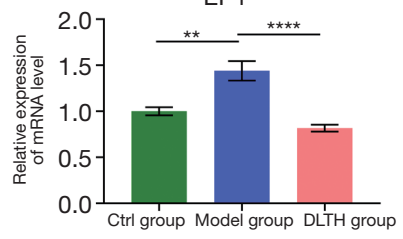

EP4
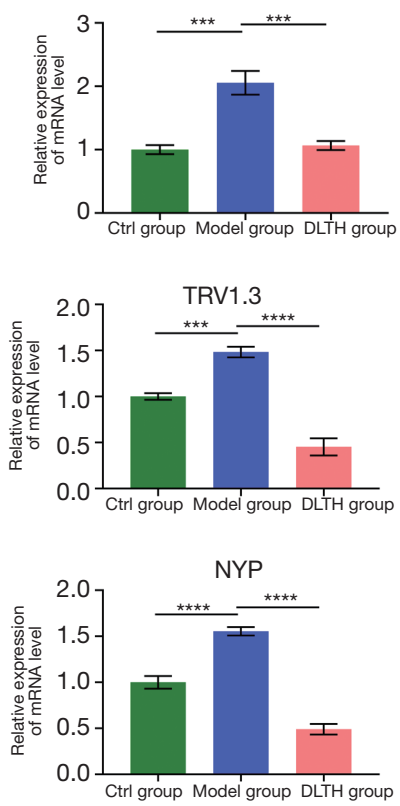

Figure 7 Pain-relieving effects of DLTH in DMM-induced osteoarthritis. (A) Effects of DLTH treatment on the NRT values in OA mice. (B) Serum level profiles of PGE2 in all groups. (C) Pain-relieving effects of DLTH on gene expressions of pro-inflammatory and pain-related factors in DRGs. All data are presented as a value relative to those in the Ctrl group. All data were expressed as mean $\pm \mathrm{SEM}$. ${ }^{\# \# \prime} \mathrm{P}<0.001$,

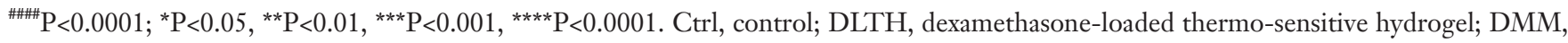
destabilization of the medial meniscus; DRGs, dorsal root ganglia; NRT, nociceptive response time; OA, osteoarthritis; PGE2, prostaglandin E2; SEM, standard error of the mean. 
counts in OA, leading to an imbalance of bone formation and resorption, and eventually a prominent reduction in subchondral bone thickness. Reduced subchondral bone thickness has been reported to be relevant to cartilage destruction $(20,27,28)$. Our study observed prominent bone loss in knee joints and subchondral bone, promoted osteolysis, significantly higher osteoclast counts, and notably lower formation of osteoblasts in the Model group. Meanwhile, decreased bone loss, suppressed osteoclast generation, and increased osteoblast formation were noted following DLTH treatment. These results signified that DLTH therapy greatly reduced the severity of bone destruction in OA.

$\mathrm{OA}$ is widely recognized as a degenerative disease with a prominent debilitating cartilage disorder. For decades, preclinical and clinical studies have primarily focused on articular cartilage degeneration. It has been reported that relatively low amounts of active MMPs are found in healthy cartilage, and thus, their contributions to aggrecan cleavage seem to be limited. However, a hallmark of OA, is cartilage proteoglycan degradation mediated by MMPs and ADAMTSs. Expressions of MMPs and ADAMTSs are both elevated in OA cartilage and activities of both MMPs and ADAMTSs are positively associated with cartilage degradation (29,30). Matrix-degrading enzymes secreted by chondrocytes (e.g., MMPs and ADAMTSs) degrade the extracellular matrix (ECM) of cartilage; these degraded ECM fragments further induce synovial inflammation and inflammatory factor generation, eventually resulting in cartilage damage and OA progression (31). In the ECM of OA cartilage, MMP-13 is the main degrading enzyme for collagen, and ADAMTS-5 is the major degrading proteinase for aggrecan $(32,33)$. For example, the increased importance of MMP-13 was demonstrated by the observation that MMP-13 overexpression in mouse cartilage resulted in proteoglycans degradation as detected by safranin $\mathrm{O}$ staining loss (29). Also, in ADAMTS-5 knock-out mice, a remarkable reduction in articular cartilage destruction in terms of proteoglycan release was found compared to wildtype mice (34).

We selected MMP-9, MMP-13, and ADAMTS-5 as our study targets among these matrix-degrading enzymes because they are widely acknowledged to play vital roles in OA. Following our animal experimental arthritis studies, $\mathrm{S} \& \mathrm{~F}$ and $\mathrm{H} \& \mathrm{E}$ staining suggested cartilage reduction and destruction in the Model group, but IA-DLTH markedly reduced cartilage pathology, as analyzed by OARSI scores. Elevated expressions of MMP-9, MMP-13, and
ADAMTS-5 were all observed in the cartilage of DMMinduced mice, whereas DLTH restrained these elevations, thereby reducing cartilage destruction. Hence, IA-DLTH may attenuate OA progression by exerting protective effects on cartilage.

Clinically, the most remarkable and disabling symptom of OA is pain (35). In our study, DLTH treatment was shown to be a promising approach for OA pain. NRT is a common indicator that reflects pain sensitivity to thermal stimuli. OA mice displayed reduced latency of hot plate nociception; however, the NRT values of the DLTH group were markedly elevated, demonstrating that OA mice exhibited pain behavior and joint injection of DLTH exerted painrelieving effects. Furthermore, in arthritis conditions, PGE2 is a well-known contributor to inflammatory pain. High levels of PGE2 are considered to induce pain and cartilage degeneration in OA (36). In our study, serum PGE2 in the Model group was notably increased, while a considerable decrease was observed in the DLTH group, which suggests that treatment with IA-DLTH eased OA arthritis pain to some degree.

Accumulating data has demonstrated that OA pain may be nociceptive, inflammatory, and neuropathic concomitantly. Arthritis pain, mediated by numerous factors responsible for both degradative and nociceptive pathways, is relevant to $\mathrm{OA}$ progression. Local inflammation in $\mathrm{OA}$ joints with the release of pro-inflammatory mediators offers a secondary contribution to pain mechanisms. Neurogenic inflammation is partly responsible for joint damage, and neuropathic pain receptors have been found in joints $(37,38)$. Injuries to joint cartilage and synovia influence peripheral afferents and DRGs, which sensitizes symptomatic pain perception via the interplay between neuropathic pathways and OA-damaged tissues. Several spinal cord pathways transmit joint nociceptive input, and inflammation potentially reduces the threshold for nociceptive stimuli. Since all of these triggers are processed via DRGs, our study targets OA pain (36).

Pro-inflammatory factors (e.g., IL- $1 \beta$, TNF- $\alpha$, IL-6, IL-17 and PGE2) induce synthesis of cartilage-degrading proteases also contribute to the development of OAassociated pain $(36,39,40)$. Pain-related cytokines (e.g., TNF- $\alpha$, IL-1 $\beta$ and IL- 6 ) are all involved in nociceptive pain in DRGs. IL- $1 \beta$ and TNF- $\alpha$ have been shown to enhance the production of PGE2, and elevated PGE2 and TNF- $\alpha$ levels coincide with other inflammatory cytokines in arthritis pain responses (41). Induced by inflammatory stimuli, COX-2 is activated and becomes a key source of 
PGE2 in locations of inflammation. The generation of PGE2 predominates in inflamed sites wherever COX-2 is active (42).

PGE2 exerts its physiological effects via EP1, EP2, EP3, and EP4 receptors. Among these, EP4 is the primary mediator that participates in OA pain symptom generation (43). Decreased pain and hypersensitivity were observed in rodents administered an EP4 prostaglandin receptor antagonist. Furthermore, Ettinger et al. found NGF activation increased PGE2 levels in a dose-dependent manner (44). NGF plays an implicated but crucial role in persistent inflammatory pain conditions and may be responsible for nociception and proalgesic effects. The pain models of Hefti's study confirmed pain sensation reduction via NGF inhibition, and other researchers have verified that the suppression of NGF or its receptors resulted in chronic inflammatory pain remission $(45,46)$. Although NGF alone is barely an inflammatory agent; however, working synergistically with other pro-inflammatory mediators worsens chronic neuropathic pain (47).

Like TRPV1, Nav1.3, and Nav1.7, Ion channel genes have also been explored in pain conduction. TRPV1 is synthesized in DRGs and is correlated to chronic inflammatory pain and peripheral neuropathy. Li et al. suggested that lower glial activity induced by inflammation or neuropathic pain was observed in TRPV1 receptorknockout mice (48). The generation of NGF enhances TRPV1-induced nociception and IL-1 $\beta$ was reported to mediate pain hypersensitivity via TRPV1 activation (49). Moreover, Nav1.3 and Nav1.7 are both promising targets in pain research, as upregulated expressions of Nav1.3 and Nav1.7 play implicit roles in neuropathic pain (50). Also, BDNF levels were correlated with pain in knee OA (51). Cruz Duarte et al. suggested that through the direct or indirect regulation of NGF, PGE2 induced BDNF expression in DRGs, thereby contributing to neuropathic and inflammatory pain genesis (52). In our study, gene expressions of IL-1 $\beta$, IL-6, IL-17, TNF- $\alpha$, COX-2, EP1, EP2, EP3, EP4, NGF, TRPV1, Nav1.3, Nav1.7, BDNF, and NYP were all notably elevated in DMMinduced DRGs, whereas OA mice receiving DLTH joint injection exhibited lower mRNA expressions of all the genes mentioned above. Taken together, these observations indicate that DLTH therapy relieved OA pain via modulation of inflammation and pain conduction processes.

DLTH enhances Dex retention time in the joint cavity, achieves high and sustained Dex concentration at the desired site, and eliminates potential adverse effects due to low-frequency therapy. All of these lead to improved therapeutic indices and fewer adverse effect risks of Dex. Thus, the resulting DDS efficiently delivers entrapped drugs over an extended period.

However, some limitations of our study have been proposed. Firstly, the $\mathrm{pH}$ used to form the gel in the release experiments should have been set to the average $\mathrm{pH}$ in the joint $(\mathrm{pH}=7.0)$. Our following research will adopt the average $\mathrm{pH}$ in the joint in the release experiments, to better improve DLTH application. Secondly, it is acknowledged that different actions of Dex occur in different stages of OA. GCs are one of the most widely used IA therapies in OA and are the standard IA management option for treating knee pain when nonpharmacological therapy, non-steroidal anti-inflammatory drugs (NSAIDS), or analgesics are not effective (53). IA administration of GCs is acknowledged to be a relatively effective, safe, adjunct conservative management. IA administration of GCs is recommended in cases of moderate to severe $\mathrm{OA}$ when patients fail to respond to NSAIDS (54). The American guidelines for treating knee OA recommend IA GCs for short-term pain relief and IA GCs combined with oral medication for better outcomes (55). In our following research, we shall pay attention to the different stages of OA. Thirdly, there are possible differences in the profiles of thermo-sensitive hydrogel between in vitro and in vivo. For example, there might be a rapid escape of a small amount of free-form Dex before gelation due to lymphatic drainage and rapid diffusion from synovial capillaries. Fourthly, hydrogels, liposomes, and nanoparticles have been considered as drug carriers that achieve a prolonged release over extended period. DDS consisting of biodegradable or biologically eliminable materials is required to avoid DDS material accumulation, which can decrease chances of inflammation or other adverse effects. Although chitosan is an ideal thermal sensitive DDS candidate for OA due to its nontoxicity, biocompatibility, biodegradability and bacterial resistance, we still observed very small soft lumps on the surface of joints after the mice were sacrificed $(56,57)$. Further exploration will be conducted on the improvement and degradation of the DLTH IA injection.

Collectively, the data presented in this study showed that IA-DLTH injections exerted chondroprotective and pain-relieving effects in DMM-induced arthritis. The involvement of MMP-9, MMP-13, ADAMTS-5, and inflammatory and pain-related factors may account for the suppression of OA progression and pain. This newly developed DLTH may represent a promising sustainable 
delivery system for cartilage protection and pain relief in the treatment of OA.

\section{Conclusions}

IA-DLTH injection exerted chondroprotective and pain-relieving effects in DMM-induced arthritis. The involvement of MMP-9, MMP-13, ADAMTS-5, and inflammatory and pain-related factors may account for the suppression of OA progression and pain. We optimized the dosage form of clinically used Dex, and this newly developed DLTH may represent a promising sustainable delivery system for cartilage protection and pain relief in the treatment of OA.

\section{Acknowledgments}

Funding: This work was supported by the National Natural Science Foundation of China (grants numbers 81874011, 81572104 and 81301531) and the Shanghai Municipal Science and Technology Commission (Innovation Grant numbers 18140903502).

\section{Footnote}

Reporting Checklist: The authors have completed the ARRIVE reporting checklist. Available at https://dx.doi. org/10.21037/atm-21-684

Data Sharing Statement: Available at https://dx.doi. org/10.21037/atm-21-684

Conflicts of Interest: All authors have completed the ICMJE uniform disclosure form (available at https://dx.doi. org/10.21037/atm-21-684). The authors have no conflicts of interest to declare.

Ethical Statement: The authors are accountable for all aspects of the work in ensuring that questions related to the accuracy or integrity of any part of the work are appropriately investigated and resolved. Experiments were performed under a project license (approval number: HKDL [2015] 16) granted by the Animal Ethical Committee of Shanghai Ninth People's Hospital, in compliance with the Code of Practice for the Housing and Care of Animals Used in Scientific Procedures and the ARRIVE (Animals in Research: Reporting In Vivo Experiments) guidelines for the care and use of animals.
Open Access Statement: This is an Open Access article distributed in accordance with the Creative Commons Attribution-NonCommercial-NoDerivs 4.0 International License (CC BY-NC-ND 4.0), which permits the noncommercial replication and distribution of the article with the strict proviso that no changes or edits are made and the original work is properly cited (including links to both the formal publication through the relevant DOI and the license). See: https://creativecommons.org/licenses/by-nc-nd/4.0/.

\section{References}

1. Hu G, Zhao X, Wang C, et al. MicroRNA-145 attenuates TNF- $\alpha$-driven cartilage matrix degradation in osteoarthritis via direct suppression of MKK4. Cell Death Dis 2017;8:e3140.

2. Tong W, Geng Y, Huang Y, et al. In Vivo Identification and Induction of Articular Cartilage Stem Cells by Inhibiting NF-кB Signaling in Osteoarthritis. Stem Cells 2015;33:3125-37.

3. Katri A, Dąbrowska A, Löfvall H, et al. A dual amylin and calcitonin receptor agonist inhibits pain behavior and reduces cartilage pathology in an osteoarthritis rat model. Osteoarthritis Cartilage 2019;27:1339-46.

4. Grodzinsky AJ, Wang Y, Kakar S, et al. Intra-articular dexamethasone to inhibit the development of posttraumatic osteoarthritis. J Orthop Res 2017;35:406-11.

5. Zhang Z, Wei X, Gao J, et al. Intra-Articular Injection of Cross-Linked Hyaluronic Acid-Dexamethasone Hydrogel Attenuates Osteoarthritis: An Experimental Study in a Rat Model of Osteoarthritis. Int J Mol Sci 2016;17:411.

6. Huebner KD, Shrive NG, Frank CB. Dexamethasone inhibits inflammation and cartilage damage in a new model of post-traumatic osteoarthritis. J Orthop Res 2014;32:566-72.

7. Zhao Y, Wei C, Chen X, et al. Drug Delivery System Based on Near-Infrared Light-Responsive Molybdenum Disulfide Nanosheets Controls the High-Efficiency Release of Dexamethasone To Inhibit Inflammation and Treat Osteoarthritis. ACS Appl Mater Interfaces 2019;11:11587-601.

8. Qi X, Qin X, Yang R, et al. Intra-articular Administration of Chitosan Thermosensitive In Situ Hydrogels Combined With Diclofenac Sodium-Loaded Alginate Microspheres. J Pharm Sci 2016;105:122-30.

9. Chen ZP, Liu W, Liu D, et al. Development of brucineloaded microsphere/thermally responsive hydrogel combination system for intra-articular administration. J 
Control Release 2012;162:628-35.

10. Wu H, Wang K, Wang H, et al. Novel self-assembled tacrolimus nanoparticles cross-linking thermosensitive hydrogels for local rheumatoid arthritis therapy. Colloids Surf B Biointerfaces 2017;149:97-104.

11. Chen M, Wu F, Pan J, et al. Preparation of Injectable Thermosensitive Meloxicam Hydrogel. China Pharmacy 2010;21:1991-3.

12. National Research Council Institute for Laboratory Animal R. Guide for the Care and Use of Laboratory Animals. Washington (DC): National Academies Press (US) Copyright 1996 by the National Academy of Sciences; 1996.

13. Kilkenny C, Browne WJ, Cuthill IC, et al. Improving bioscience research reporting: the ARRIVE guidelines for reporting animal research. PLoS Biol 2010;8:e1000412.

14. Zhou F, Mei J, Yuan K, et al. Isorhamnetin attenuates osteoarthritis by inhibiting osteoclastogenesis and protecting chondrocytes through modulating reactive oxygen species homeostasis. J Cell Mol Med 2019;23:4395-407.

15. Nixon AJ, Grol MW, Lang HM, et al. Disease-Modifying Osteoarthritis Treatment With Interleukin-1 Receptor Antagonist Gene Therapy in Small and Large Animal Models. Arthritis Rheumatol 2018;70:1757-68.

16. Fan KJ, Wu J, Wang QS, et al. Metformin inhibits inflammation and bone destruction in collagen-induced arthritis in rats. Ann Transl Med 2020;8:1565.

17. Glasson SS, Chambers MG, Van Den Berg WB, et al. The OARSI histopathology initiative - recommendations for histological assessments of osteoarthritis in the mouse. Osteoarthritis Cartilage 2010;18 Suppl 3:S17-23.

18. Wang Q, Xu B, Fan K, et al. Inflammation suppression by dexamethasone via inhibition of CD147-mediated NF$\kappa \mathrm{B}$ pathway in collagen-induced arthritis rats. Mol Cell Biochem 2020;473:63-76.

19. Wang Q, Xu B, Fan K, et al. CypB-CD147 Signaling Is Involved in Crosstalk between Cartilage and FLS in Collagen-Induced Arthritis. Mediators Inflamm 2020;2020:6473858.

20. Zhou F, Mei J, Han X, et al. Kinsenoside attenuates osteoarthritis by repolarizing macrophages through inactivating $\mathrm{NF}-\kappa \mathrm{B} / \mathrm{MAPK}$ signaling and protecting chondrocytes. Acta Pharm Sin B 2019;9:973-85.

21. Chang TK, Wang YH, Kuo SJ, et al. Apelin enhances IL$1 \beta$ expression in human synovial fibroblasts by inhibiting miR-144-3p through the PI3K and ERK pathways. Aging (Albany NY) 2020;12:9224-39.
22. Liao L, Zhang S, Zhao L, et al. Acute Synovitis after Trauma Precedes and is Associated with Osteoarthritis Onset and Progression. Int J Biol Sci 2020;16:970-80.

23. Woo CH, Kim HK, Jung GY, et al. Small extracellular vesicles from human adipose-derived stem cells attenuate cartilage degeneration. J Extracell Vesicles 2020;9:1735249.

24. Wu X, Wang Y, Xiao Y, et al. Extracellular vesicles: Potential role in osteoarthritis regenerative medicine. J Orthop Translat 2020;21:73-80.

25. Pessler F, Ogdie A, Diaz-Torne C, et al. Subintimal $\mathrm{Ki}-67$ as a synovial tissue biomarker for inflammatory arthropathies. Ann Rheum Dis 2008;67:162-7.

26. Berenbaum F, Walker C. Osteoarthritis and inflammation: a serious disease with overlapping phenotypic patterns. Postgrad Med 2020;132:377-84.

27. Burr DB, Gallant MA. Bone remodelling in osteoarthritis. Nat Rev Rheumatol 2012;8:665-73.

28. Findlay DM, Kuliwaba JS. Bone-cartilage crosstalk: a conversation for understanding osteoarthritis. Bone Res 2016;4:16028.

29. van Geffen EW, van Caam APM, Schreurs W, et al. IL-37 diminishes proteoglycan loss in human OA cartilage: donor-specific link between IL-37 and MMP-3. Osteoarthritis Cartilage 2019;27:148-57.

30. Xu K, Ma C, Xu L, et al. Polygalacic acid inhibits MMPs expression and osteoarthritis via $\mathrm{Wnt} / \beta$-catenin and MAPK signal pathways suppression. Int Immunopharmacol 2018;63:246-52.

31. Sirikaew N, Chomdej S, Tangyuenyong S, et al. Proinflammatory cytokines and lipopolysaccharides up regulate MMP-3 and MMP-13 production in Asian elephant (Elephas maximus) chondrocytes: attenuation by anti-arthritic agents. BMC Vet Res 2019;15:419.

32. Feng X, Pan J, Li J, et al. Metformin attenuates cartilage degeneration in an experimental osteoarthritis model by regulating AMPK/mTOR. Aging (Albany NY) 2020;12:1087-103.

33. Jung YK, Park HR, Cho HJ, et al. Degrading products of chondroitin sulfate can induce hypertrophy-like changes and MMP-13/ADAMTS5 production in chondrocytes. Sci Rep 2019;9:15846.

34. Majumdar MK, Askew R, Schelling S, et al. Doubleknockout of ADAMTS-4 and ADAMTS-5 in mice results in physiologically normal animals and prevents the progression of osteoarthritis. Arthritis Rheum 2007;56:3670-4.

35. Hunter DJ, McDougall JJ, Keefe FJ. The symptoms of 
osteoarthritis and the genesis of pain. Rheum Dis Clin North Am 2008;34:623-43.

36. Lee AS, Ellman MB, Yan D, et al. A current review of molecular mechanisms regarding osteoarthritis and pain. Gene 2013;527:440-7.

37. Perrot S. Osteoarthritis pain. Best Pract Res Clin Rheumatol 2015;29:90-7.

38. Dieppe PA, Lohmander LS. Pathogenesis and management of pain in osteoarthritis. Lancet 2005;365:965-73.

39. Nencini S, Ringuet M, Kim DH, et al. Mechanisms of nerve growth factor signaling in bone nociceptors and in an animal model of inflammatory bone pain. Mol Pain 2017;13:1744806917697011.

40. Lee YM, Son E, Kim SH, et al. Anti-inflammatory and anti-osteoarthritis effect of Mollugo pentaphylla extract. Pharm Biol 2019;57:74-81.

41. Yu HM, Wang Q, Sun WB. Silencing of FKBP51 alleviates the mechanical pain threshold, inhibits DRG inflammatory factors and pain mediators through the NFkappaB signaling pathway. Gene 2017;627:169-75.

42. Kirkby Shaw K, Rausch-Derra LC, Rhodes L. Grapiprant: an EP4 prostaglandin receptor antagonist and novel therapy for pain and inflammation. Vet Med Sci 2016;2:3-9.

43. Li X, Ellman M, Muddasani P, et al. Prostaglandin E2 and its cognate EP receptors control human adult articular cartilage homeostasis and are linked to the pathophysiology of osteoarthritis. Arthritis Rheum 2009;60:513-23.

44. Ettinger K, Nevo Y, Marcinkiewicz C, et al. Nerve growth factor-induced myoprotection in $\mathrm{C} 2 \mathrm{C} 12$ muscle cells is mediated by $\alpha 9 \beta 1$ integrin via release of PGE2. J Basic Clin Physiol Pharmacol 2015;26:411-5.

45. Hefti FF, Rosenthal A, Walicke PA, et al. Novel class of pain drugs based on antagonism of NGF. Trends Pharmacol Sci 2006;27:85-91.

46. Miyagi M, Ishikawa T, Kamoda H, et al. Efficacy of nerve growth factor antibody in a knee osteoarthritis pain model in mice. BMC Musculoskelet Disord 2017;18:428.

47. Ashraf S, Bouhana KS, Pheneger J, et al. Selective inhibition of tropomyosin-receptor-kinase A (TrkA) reduces pain and joint damage in two rat models of inflammatory arthritis. Arthritis Res Ther 2016;18:97.

48. Li X, Kroin JS, Kc R, et al. Altered spinal microRNA-146a and the microRNA-183 cluster contribute to osteoarthritic pain in knee joints. J Bone Miner Res 2013;28:2512-22.
49. Chen Y, Willcockson HH, Valtschanoff JG. Influence of the vanilloid receptor TRPV1 on the activation of spinal cord glia in mouse models of pain. Exp Neurol 2009;220:383-90.

50. Xie MX, Yang J, Pang RP, et al. Bulleyaconitine A attenuates hyperexcitability of dorsal root ganglion neurons induced by spared nerve injury: The role of preferably blocking Nav1.7 and Nav1.3 channels. Mol Pain 2018;14:1744806918778491.

51. Simão AP, Mendonça VA, de Oliveira Almeida TM, et al. Involvement of BDNF in knee osteoarthritis: the relationship with inflammation and clinical parameters. Rheumatol Int 2014;34:1153-7.

52. Cruz Duarte P, St-Jacques B, Ma W. Prostaglandin E2 contributes to the synthesis of brain-derived neurotrophic factor in primary sensory neuron in ganglion explant cultures and in a neuropathic pain model. Exp Neurol 2012;234:466-81.

53. Jones IA, Togashi R, Wilson ML, et al. Intra-articular treatment options for knee osteoarthritis. Nat Rev Rheumatol 2019;15:77-90.

54. Savvidou O, Milonaki M, Goumenos S, et al. Glucocorticoid signaling and osteoarthritis. Mol Cell Endocrinol 2019;480:153-66.

55. Löwenberg M, Stahn C, Hommes DW, et al. Novel insights into mechanisms of glucocorticoid action and the development of new glucocorticoid receptor ligands. Steroids 2008;73:1025-9.

56. Ji QX, Zhao QS, Deng J, et al. A novel injectable chlorhexidine thermosensitive hydrogel for periodontal application: preparation, antibacterial activity and toxicity evaluation. J Mater Sci Mater Med 2010;21:2435-42.

57. Shamji MF, Hwang P, Bullock RW, et al. Release and activity of anti-TNFalpha therapeutics from injectable chitosan preparations for local drug delivery. J Biomed Mater Res B Appl Biomater 2009;90:319-26.

(English Language Editors: A. Kassem and J. Chapnick)

Cite this article as: Wang QS, Xu BX, Fan KJ, Fan YS, Teng H, Wang TY. Dexamethasone-loaded thermo-sensitive hydrogel attenuates osteoarthritis by protecting cartilage and providing effective pain relief. Ann Transl Med 2021;9(14):1120. doi: 10.21037/atm-21-684 